\title{
Dispersive sensing in hybrid InAs/Al nanowires
}

Sabonis, Deividas; O'Farrell, Eoin C. T.; Razmadze, Davydas; van Zanten, David M. T.; Suter, Judith; Krogstrup, Peter; Marcus, Charles M.

Published in:

Applied Physics Letters

DOI:

$10.1063 / 1.5116377$

Publication date:

2019

Document version

Publisher's PDF, also known as Version of record

Citation for published version (APA):

Sabonis, D., O'Farrell, E. C. T., Razmadze, D., van Zanten, D. M. T., Suter, J., Krogstrup, P., \& Marcus, C. M. (2019). Dispersive sensing in hybrid InAs/Al nanowires. Applied Physics Letters, 115(10), [102601]. https://doi.org/10.1063/1.5116377 


\section{Dispersive sensing in hybrid InAs/Al nanowires}

Cite as: Appl. Phys. Lett. 115, 102601 (2019); https://doi.org/10.1063/1.5116377

Submitted: 25 June 2019 . Accepted: 25 July 2019 . Published Online: 03 September 2019

Deividas Sabonis (D), Eoin C. T. O'Farrell, Davydas Razmadze, David M. T. van Zanten, Judith Suter, Peter Krogstrup, and Charles M. Marcus

\section{COLLECTIONS}

F This paper was selected as Featured
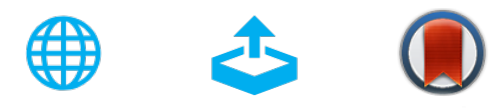

\section{ARTICLES YOU MAY BE INTERESTED IN}

A quantum engineer's guide to superconducting qubits

Applied Physics Reviews 6, 021318 (2019); https://doi.org/10.1063/1.5089550

Strain engineering in $\mathrm{Ge} / \mathrm{GeSn}$ core/shell nanowires

Applied Physics Letters 115, 113102 (2019); https://doi.org/10.1063/1.5111872

Reversible thermal diode and energy harvester with a superconducting quantum interference single-electron transistor

Applied Physics Letters 115, 092601 (2019); https://doi.org/10.1063/1.5109100

\section{Lock-in Amplifiers up to $600 \mathrm{MHz}$}
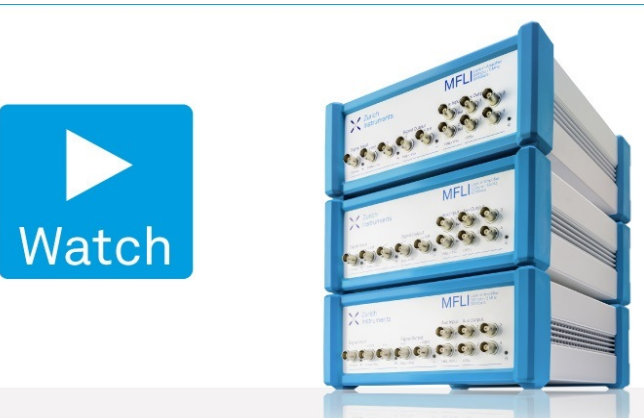


\title{
Dispersive sensing in hybrid InAs/Al nanowires
}

\author{
Cite as: Appl. Phys. Lett. 115, 102601 (2019); doi: 10.1063/1.5116377 \\ Submitted: 25 June 2019 . Accepted: 25 July 2019 .
}

Published Online: 3 September 2019
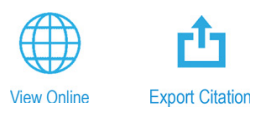

Deividas Sabonis, ${ }^{1, a)}$ (D) Eoin C. T. O'Farrell, ${ }^{1, a)}$ Davydas Razmadze, David M. T. van Zanten, Judith Suter, Peter Krogstrup, ${ }^{2}$ and Charles M. Marcus ${ }^{1, b)}$

\author{
AFFILIATIONS \\ Universitetsparken 5, 2100 Copenhagen, Denmark \\ 2800 Kongens Lyngby, Denmark \\ a) Contributions: D. Sabonis and E. C. T. O'Farrell contributed equally to this work. \\ ${ }^{b)}$ Electronic mail: marcus@nbi.ku.dk
}

${ }^{7}$ Microsoft Quantum Lab Copenhagen and Center for Quantum Devices, Niels Bohr Institute, University of Copenhagen,

${ }^{2}$ Microsoft Quantum Materials Lab and Center for Quantum Devices, Niels Bohr Institute, University of Copenhagen, Kanalvej 7,

\begin{abstract}
Dispersive charge sensing is realized in hybrid semiconductor-superconductor nanowires in gate-defined single- and double-island device geometries. Signal-to-noise ratios (SNRs) were measured in both the frequency and time domains. Frequency-domain measurements were carried out as a function of frequency and power and yield a charge sensitivity of $1 \times 10^{-3} \mathrm{e} / \sqrt{\mathrm{Hz}}$ for an $\sim 11 \mathrm{MHz}$ measurement bandwidth. Time-domain measurements yield SNR $>1$ for a $20 \mu$ s integration time. At zero magnetic field, photon-assisted tunneling was detected dispersively in a double-island geometry, indicating coherent hybridization of the two superconducting islands. At an axial magnetic field of $0.6 \mathrm{~T}$, subgap states are detected dispersively, demonstrating the suitability of the method to sensing in the topological regime.
\end{abstract}

Published under license by AIP Publishing. https://doi.org/10.1063/1.5116377

Readout of quantum systems on timescales short compared to coherence or relaxation times is typically performed by one of a few schemes: (i) the device is incorporated into a resonant circuit, allowing state-dependent changes in the damping or shift of the resonance to be measured, ${ }^{1-4}$ (ii) the quantum state is converted to charge, which is then detected by a nearby electrometer, ${ }^{5-8}$ or (iii) a state-dependent capacitive coupling to the system results in a frequency shift in the coupled resonant circuit that depends on the quantum state, ${ }^{9,10}$ the latter referred to as dispersive readout. In the context of topological qubits, several proposals for nonlocally encoding fermion parity in Majorana zero modes have been made. ${ }^{11-15}$ Some proposals use parity-to-charge conversion for readout, ${ }^{12}$ while others use statedependent hybridization of the Majorana mode with an ancillary system, leading to a dispersive readout signal. ${ }^{13,14}$

Integrating readout circuitry into an existing electrostatic gate or ohmic contact is useful for reducing the device footprint and lead count. ${ }^{10,16-27}$ In this case, dispersive readout is performed by monitoring state-dependent shifts in the resonance frequency $f_{\mathrm{R}}=\left(L C_{\text {tot }}\right)^{-1 / 2}$ of an $L C$ circuit connected to a gate, where $f_{\mathrm{R}}$ is detuned from the qubit transition frequency. The total capacitance, $C_{\text {tot }}$, comprises geometric capacitance, $C_{g}$ (including parasitic contributions), quantum capacitance, $C_{\mathrm{Q}}$, and tunnel capacitance, $C_{\mathrm{T}} \cdot{ }^{9,28}$ When the quantum system consists of a Coulomb island tunnel coupled to a reservoir, $C_{\mathrm{Q}}$ arises from continuous charge transitions and is proportional to the curvature of energy with respect to the confining gate voltage. ${ }^{29}$ The maximum magnitude of $C_{\mathrm{Q}}$ occurs at gate voltages corresponding to charge degeneracy, with opposite signs for ground and first excited states. $C_{\mathrm{T}}$ is significant when the energy relaxation rate exceeds $f_{\mathrm{R}}$. The dependence of $f_{\mathrm{R}}$ on $C_{\mathrm{Q}}$ provides the quantum state selectivity of the dispersive shift. Monitoring phase or magnitude of the signal reflected from the resonant circuit thus allows readout of the quantum state of the system.

Recent work on gate-based dispersive sensing has addressed semiconducting nanowires (NWs) ${ }^{30,31}$ and semiconductor quantum dots coupled to subgap states in semiconductor-superconductor NWs at zero magnetic field. ${ }^{32}$ Beyond qubit readout, dispersive sensing has been used to allow rapid tuning of quantum devices, yielding complementary information to conventional transport approaches. ${ }^{3}$

In this letter, we explore dispersive charge sensing in an epitaxial semiconductor-superconductor (InAs/Al) nanowire device configured to form either a single or double island depending on gate voltages. Since both proximity-induced superconductivity and high magnetic fields are needed for realizing the topological regime, we have focused particular attention on operation at magnetic fields compatible with topological superconductivity. At zero field, we extract a signal-tonoise ratio (SNR) of a gate-based dispersive sensor as a measure of its 
sensitivity. Time-domain measurements gave SNR $>1$ for integration times $>20 \mu \mathrm{s}$, as described in detail below. Applying a continuous microwave drive to a nearby gate induces photon assisted tunneling (PAT),${ }^{34,35}$ indicating coherent hybridization of the two islands.

The device, shown in Fig. 1(a), is based on an InAs/Al NW with $7 \mathrm{~nm}$ of epitaxial $\mathrm{Al}$ grown on three facets of the hexagonal cross section. ${ }^{36}$ Following deposition of individual wires on a Si-SiOx substrate, three $100 \mathrm{~nm}$ segments of $\mathrm{Al}$ were removed by wet etching to provide tunable tunnel barriers. An insulating layer of $\mathrm{HfO}_{2}$ was then deposited over the wire and electrostatic gates labeled C1, C2, C3, and C4 were deposited, creating three segments of lengths $\sim 2.5 \mu \mathrm{m}, 1 \mu \mathrm{m}$, and $3.5 \mu \mathrm{m}$, separated by gate-voltage-controlled barriers of InAs only (see the supplementary material for fabrication details). The detector gate, labeled P2, and the right ohmic contact were bonded to superconducting spiral inductors ${ }^{23}$ fabricated on a separate sapphire chip to form a resonant circuit that was capacitively coupled to a conventional radio frequency (rf) reflectometry detection chain. Each resonator was also connected to bias resistors, allowing DC voltages to be applied to the gates. Data from two devices (D1 and D2) are presented. All measurements were made in a dilution refrigerator with a base temperature of $\sim 20 \mathrm{mK}$ equipped with a vector magnet (see the supplementary material for measurement details).

We initially consider zero magnetic field. Electrostatic gates C2 and $\mathrm{C} 3$ were set to the tunneling regime, forming a superconducting single island. A comparison of conductance measured via lowfrequency transport using a lock-in amplifier vs reflectometry from the right ohmic contact is shown in Fig. 1(b). The conductance, $g$, around zero bias showed Coulomb blockade (CB) peaks as a function of plunger voltage $V_{\mathrm{P} 2}$. Finite bias conductance measurements yielded a charge energy of $E_{\mathrm{C}}=60 \mu \mathrm{eV}$. CB oscillations had a period of two electron (2e) charge. This is expected for $\Delta>E_{\mathrm{C}}$, where $\Delta \sim 180 \mu \mathrm{eV}$ is the induced superconducting gap and indicates low average quasiparticle poisoning. Figure 1(c) shows the ohmic reflectometer response as a function of plunger voltage $V_{\mathrm{P} 2}$ and $\mathrm{rf}$ readout frequency $f_{\mathrm{rf}}$, see the supplementary material for details. The resonance shows a statedependent shift when crossing the island degeneracy [Fig. 1(d)]. As shown later, PAT was observed at frequencies $\geq 11 \mathrm{GHz}$, i.e., highly
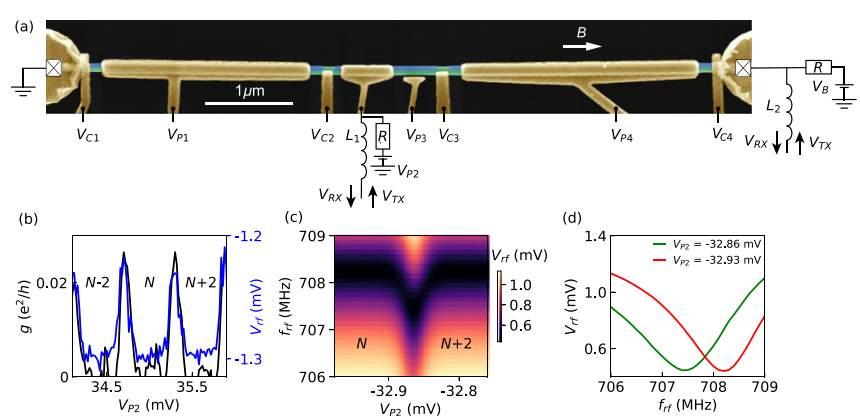

FIG. 1. (a) Scanning electron micrograph of the nanowire device together with the relevant electrostatic gates labeled. (b) Coulomb blockade oscillations of the superconducting single-island defined between gates $\mathrm{C} 2$ and $\mathrm{C} 3$ as a function of plunger gate voltage $V_{\mathrm{P} 2}$ recorded in conductance $g$ via lock-in transport (black) and dispersive lead sensing $V_{\text {rf }}$ (blue) showing close matching. (c) Lead sensor signal $V_{\text {rf }}$ as a function of electrostatic plunger gate voltage $V_{P 2}$ tuning the superconducting island over the Coulomb degeneracy as a function of readout frequency $f_{\mathrm{rf}}$ (d) Line cuts from (c) on- (green) and off- (red) Coulomb degeneracy. Dispersive shift in frequency can be observed as the island is tuned over the degeneracy. detuned from the readout resonators, indicating a state-dependent dispersive interaction between the resonator and the device.

In a lithographically similar device (D2), the gate sensor sensitivity at zero magnetic field was evaluated in a superconducting singleisland regime, and the island had charging energy $E_{\mathrm{C}}=105 \mu \mathrm{eV}$. A nearby gate [P3 in Fig. 1(a)] was modulated with a sinusoidal signal of fixed frequency $\left(f_{\mathrm{M}}\right)$ and amplitude $V_{\mathrm{M}} \cdot{ }^{8,10,22}$ Positioning the island gate $V_{\mathrm{P} 2}$ on the side of a $\mathrm{CB}$ peak amplitude modulates the island charge and thereby the readout resonance, inducing sidebands that are symmetrically detuned by $f_{\mathrm{M}}$ from the carrier frequency, $f_{\mathrm{rf}}$.

Figure 2(a) shows the signal reflected from the detector gate P2 recorded with a spectrum analyser with $\Delta f=13.4 \mathrm{~Hz}$ resolution bandwidth. Around the resonance frequency $(\sim 438 \mathrm{MHz})$, two sidebands are observed at $f_{\mathrm{M}}= \pm 12 \mathrm{kHz}$. For the analysis that follows, the upper sideband was chosen. The signal-to-noise ratio (SNR) is given by the ratio of the height of the sideband to the noise floor in a given bandwidth. The SNR dependence on the rf carrier power $P_{\mathrm{rf}}$ (before $\sim 40 \mathrm{~dB}$ attenuation) is shown in Fig. 2(b). An initial increase in SNR up to around $P_{\mathrm{rf}}=-35 \mathrm{dBm}$ is observed followed by a decrease for larger power. This turnaround behavior can be explained by the tradeoff between lifetime and power-induced broadening. ${ }^{19}$ Figure 2(c) shows the SNR dependence on $f_{\mathrm{rf}}$ for a fixed $P_{\mathrm{rf}}=-35 \mathrm{dBm}$. A maximum SNR was observed at $f_{\mathrm{rf}} \sim 435 \mathrm{MHz}$. The full-width half-maximum of the SNR as a function of $f_{\mathrm{rf}}$ indicates an approximate resonator bandwidth of $\sim 12.2 \mathrm{MHz}$.

With $P_{\mathrm{rf}}$ and $f_{\mathrm{rf}}$ set to maximize SNR [see Figs. 2(b) and 2(c)], a detection bandwidth of $\sim 11 \mathrm{MHz}$ was determined ${ }^{10}$ by measuring the modulation frequency $f_{\mathrm{M}}$ at which SNR decreased by $3 \mathrm{~dB}$, as shown in Fig. 2(d). We next evaluate the charge sensitivity $S \equiv \Delta q(2 \Delta f)^{-1 / 2}$ $10^{-\mathrm{SNR} / 20}$, measured in the time domain, ${ }^{37}$ taking spectral resolution $\Delta f=13.4 \mathrm{~Hz}$ and $\mathrm{SNR} \sim 15$ as a typical value for optimal detection parameters [see Figs. 2(b) and 2(c)]. The effective charge change induced (a)
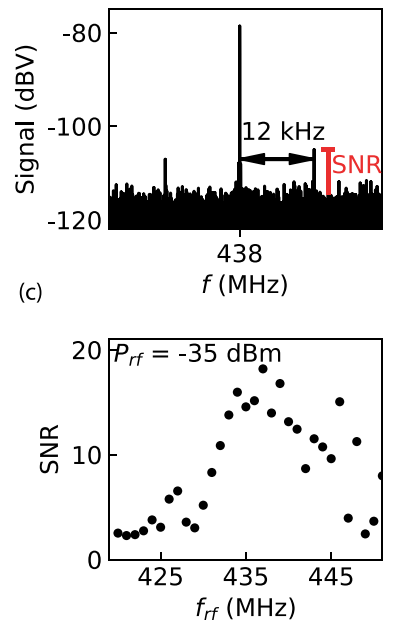

(b)

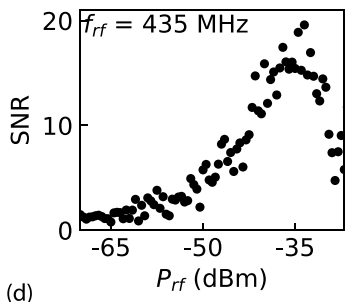

(d)

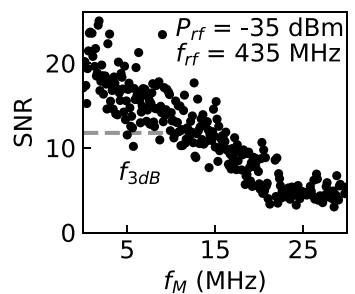

FIG. 2. (a) Spectrum reflected from the detector gate $P 2$ under gate-modulation as described in the main text; two sidebands symmetrically detuned from resonance were observed. (b) Signal-to-noise ratio dependence on carrier power $P_{\mathrm{rf}}$ and (c) on carrier frequency $f_{\text {rf. }}$ (d) Signal-to-noise ratio as a function of modulation frequency $f_{\mathrm{M}}$. The $3 \mathrm{~dB}$ frequency, $\sim 11 \mathrm{MHz}$, indicates the bandwidth of the measurement. 
by modulation at amplitude $V_{\mathrm{M}}=30 \mathrm{~m} \mathrm{~V} \mathrm{~V}_{\mathrm{pp}}$ was $\Delta q=e\left(30 \mathrm{mV}_{\mathrm{pp}} /\right.$ $\left.950 \mathrm{mV}_{\mathrm{pp}}\right) \sim 0.03 e$, found by comparing the amplitude of $V_{\mathrm{M}}(30 \mathrm{~m}$ $\mathrm{V}_{\mathrm{pp}}$ ) to the amplitude needed to sweep over a full CB peak spacing $(950 \mathrm{~m}$ $\mathrm{V}_{\mathrm{pp}}$ ). The factor $1 / \sqrt{2}$ accounts for the power collected from both sidebands. The resulting charge sensitivity was $S \sim 1 \times 10^{-3} \mathrm{e} / \sqrt{\mathrm{Hz}}$.

The time needed to obtain a particular SNR at optimal values of $f_{\mathrm{rf}}=438 \mathrm{MHz}$ and $P_{\mathrm{rf}}=-40 \mathrm{dBm}$ was determined by comparing the difference, $\delta V$, in signal $V_{\mathrm{rf}}$ on and off a CB peak with the noise of the measurement, which decreased with increasing signal averaging. In the single-island regime, gate $\mathrm{P} 2$ was pulsed on and off a CB peak with amplitude equivalent to $\sim 0.3 e$ at a repetition frequency of $2 \mathrm{kHz}$. Inphase $(I)$ and quadrature $(Q)$ components were recorded with a time constant of $500 \mathrm{~ns}$, averaged over an integration time $\tau$, and plotted in the complex $I$ - Q plane. Figure 3(a) shows an example with $\sim 1.1$ $\times 10^{4}$ points averaged for $\tau=20 \mu$ s each. SNR $(\tau)$ was found by fitting to two 2D Gaussians, yielding signal $\delta V$ and noise, $2 \sigma$. The timedomain SNR, given by $\delta V / 2 \sigma(\tau)$, is shown in Fig. $3(\mathrm{~b})$. SNR $\sim 1$ is reached for an integration time of $\tau \sim 20 \mu$ s.

Figure 4(a) shows $V_{\text {rf }}$ measured using a dispersive sensor on gate P2 [see Fig. 1(a)] rather than an ohmic contact. Coulomb diamonds were observed with the device configured as single island at $B=0$. Each measured point in Fig. 4 was averaged 100 times, yielding a measurement time of $60 \mu$ s per point. At high bias, $V_{B}>0.2 \mathrm{mV}$, the period of Coulomb oscillations was halved compared to low bias, indicating that low-bias transport was predominantly carried by Cooper pairs. Figure 4(b) shows the phase response of the demodulated signal. The readout frequency was chosen to optimize the $1 e$ charge transitions leading to a nonmonotonic response for $2 e$ charge transitions that had a larger capacitive shift of the resonator. The estimated gate sensor geometric capacitance $C_{\mathrm{g}}=e / \delta V_{\mathrm{g}} \sim 0.55 \mathrm{fF}$, where $\delta V_{\mathrm{g}}$ is a gate space periodicity of the island plunger P2. This makes $C_{\mathrm{g}} \sim 0.4 C_{\Sigma}$ using the normal-state charging energy of the island.

Application of a parallel magnetic field, $B$, induced subgap states in the nanowire and an evolution from $2 e$ to $1 e$ periodic CB oscillations, as shown in Fig. 4 (c), where an average $V_{\text {rf }}$ was subtracted at each field. $1 e$ periodic $\mathrm{CB}$ oscillations correspond to a state at zeroenergy in the superconducting gap, ${ }^{38}$ though not necessarily a discrete state. To keep maximum detection contrast at each magnetic field value, the readout frequency was adjusted to compensate for changing kinetic inductance of the resonator. The jump at $B \sim 0.4 \mathrm{~T}$ was likely due to electrostatic background charges in the NW environment. The detected signal did not degrade at magnetic field ranges compatible with tuning into a topological state in similar wires.
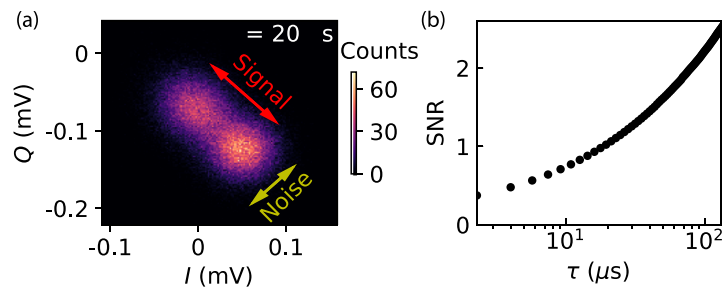

FIG. 3. (a) Two distinguishable states indicated in in-phase $(I)$ and quadrature $(Q)$ planes together with "Signal" $\delta V$ defined as a separation between two IQ space maxima and "Noise" $2 \sigma$ as induced broadening of the two maxima. (b) Signal-tonoise ratio $(\delta V / 2 \sigma)$ as a function of integration time $\tau$. (a)

(b)
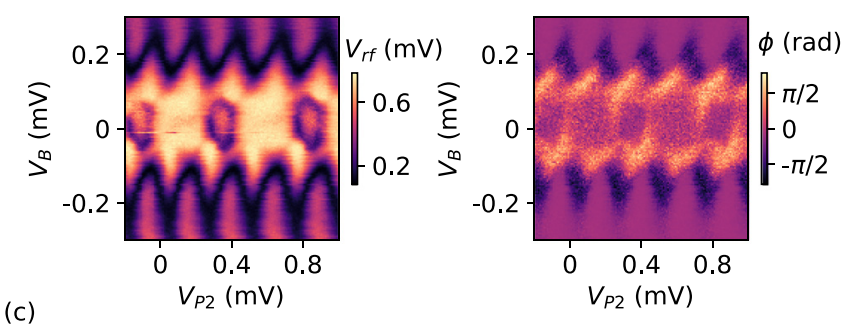

(c)

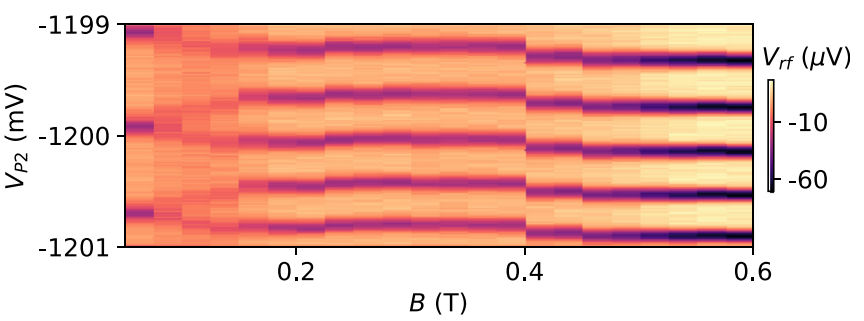

FIG. 4. (a) Gate P2 sensing of Coulomb blockaded superconducting single-island recorded in magnitude $V_{\mathrm{rf}}(\mathrm{a})$ and phase $\phi$ (b) of the demodulated signal as a function of island plunger voltage $V_{P 2}$ and bias $V_{B}$. (c) Coulomb blockade evolution from the $2 e$ to 1 e periodic regime as a function of parallel to the nanowire axis magnetic field $B$ and island plunger voltage $V_{\mathrm{P} 2}$ (line average along the $V_{\mathrm{P} 2}$ axis subtracted).

The gate voltage $V_{P 2}$ for Fig. 4(c) was selected based on the appearance of a discrete state in the spectrum at a high magnetic field, whereas Fig. 4(a) is at zero magnetic field where subgap states are not present. Previous studies ${ }^{39}$ have shown that in hybrid InAs/Al nanowires, the potential should be tuned to induce a discrete state in the subgap spectrum of the nanowire.

The lifetime of the excited state was too short to measure directly but could be estimated using PAT in a double-dot configuration. At zero field, the $2 e$-periodic double-dot charge stability diagram as a function of voltages $V_{\mathrm{P} 2}$ and $V_{P 4}$ takes on a familiar honeycomb pattern ${ }^{40-42}$ as seen in Fig. 5(a). The gate sensor detected two types of transitions: internal, between the two islands, and external, to the left superconducting lead. Transitions to the right normal lead could also be discerned, though with less visibility. Spectroscopy was performed by irradiating the sample with microwaves of frequency $f_{d}$. When $f_{d}$ was comparable to or larger than the tunneling rate of Cooper pairs across the junction, $h f_{d}>E_{J}$, where $E_{J}$ is the Josephson energy, characteristic PAT features were observed in the charge stability diagram ${ }^{40}$ as seen in Fig. 5(b). As usual, ${ }^{35,41,43}$ PAT signatures appear as parallel features along the detuning axis. To quantify the interdot coupling, $f_{d}$ was swept with the system tuned using $\mathrm{P} 4$ to cross the interdot charge transitions when gate $\mathrm{P} 2$ was also swept. The resulting 2D plot, shown in Fig. 5(c), reveals the emergence of PAT features above $11.3 \mathrm{GHz}$, placing a rough lower bound on $E_{5}$. This extends our previous work on PAT in hybrid doubledot systems ${ }^{35}$ to the technique of dispersive readout.

Finally, we note that the detection sensitivity presented here is comparable to previous reports of gate-based sensing. For example, using similar inductors, Colless et al. report a charge sensitivity of $6.3 \times 10^{-3} \mathrm{e} / \sqrt{\mathrm{Hz}}$ with a $10 \mathrm{MHz}$ detection bandwidth, compatible with our result. ${ }^{10}$ However, we do not reach the highest sensitivities realized using gate-based sensing in nanowire transistors ${ }^{19,24}$ $\left(37 \times 10^{-6} \mathrm{e} / \sqrt{\mathrm{Hz}}\right.$ and $1.3 \times 10^{-6} \mathrm{e} / \sqrt{\mathrm{Hz}}$, respectively $)$, rf-quantum 


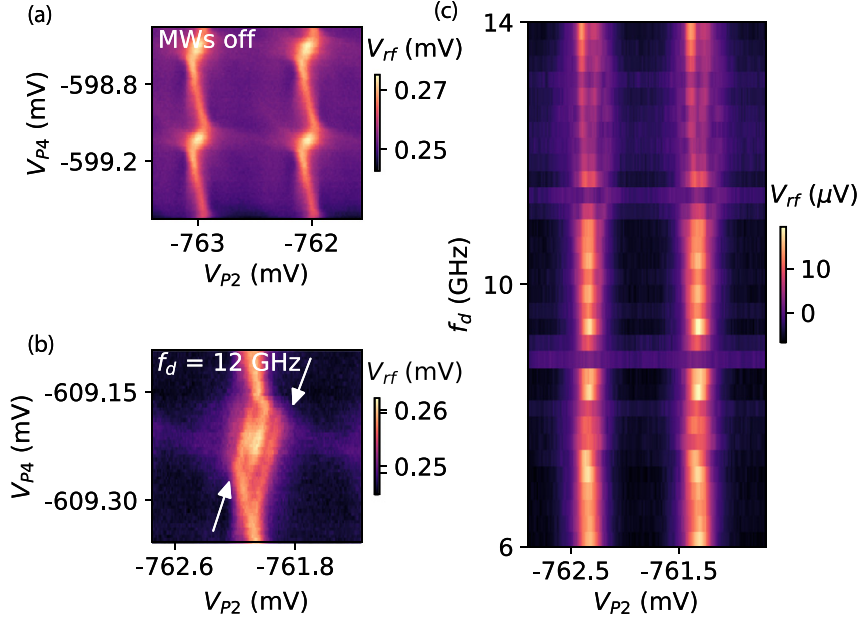

FIG. 5. (a) 2e periodic charge stability diagram as a function of right $\left(V_{P 4}\right)$ and middle plunger $\left(V_{\mathrm{P}_{2}}\right)$ voltages. (b) Microwave induced photon assisted tunneling transitions between two charge states in a double-island at zero magnetic field. (c) Energy dispersion of the double-island energy levels measured as a function of the plunger voltage $V_{\mathrm{P} 2}$ and microwave drive frequency $f_{d}$.

point $\operatorname{contacts}^{3}\left(2 \times 10^{-4} \mathrm{e} / \sqrt{\mathrm{Hz}}\right)$, or rf-SETs ${ }^{1,44}$ with typical sensitivities $1 \times 10^{-5} \mathrm{e} / \sqrt{\mathrm{Hz}}$. The latest results from silicon spin qubits using gate sensing ${ }^{18}$ reach a sensitivity of $4.1 \times 10^{-4} \mathrm{e} / \sqrt{\mathrm{Hz}}$ with an estimated $\mathrm{SNR}=6$ for a $1 \mu$ s integration time. In our case, sensitivity could be further improved by, for example, decreasing the parasitic capacitance. The results will be useful in employing gate and leadbased dispersive sensing in topological qubit experiments without the requirement of fabricating nearby electrometers.

In conclusion, gate and lead-based dispersive sensing techniques were applied to Coulomb blockaded single- and double-islands in hybrid semiconductor-superconductor InAs/Al nanowires. Characterization of gate sensing, using sideband modulation, at zero magnetic field, as a function of readout parameters, yielded charge sensitivities of the order of $1 \times 10^{-3} \mathrm{e} / \sqrt{\mathrm{Hz}}$, with a detection bandwidth of $\sim 11 \mathrm{MHz}$. In time-domain measurements, SNR of 1 was achieved for an integration time of $20 \mu \mathrm{s}$. Dispersive readout of photon assisted tunneling indicated that coherent hybridization of two superconducting islands gave an estimate for the Josephson coupling between islands, $E_{J} \sim 11.3 \mathrm{GHz}$. Magnetic field compatibility of the gate sensor up to $0.6 \mathrm{~T}$ was demonstrated, compatible with tuning into the topological regime.

See the supplementary material for specific information regarding parameters of the resonant circuit and measurement techniques used and device fabrication methods.

We thank Shivendra Upadhyay for help with fabrication and Ruben Grigoryan, Andrew Higginbotham, John Hornibrook, Ferdinand Kuemmeth, Wolfgang Pfaff, Bernard van Heck, and John Watson for valuable discussions. This research was supported by Microsoft and the Danish National Research Foundation. P.K. acknowledges support from the ERC starting Grant No. 716655 under the Horizon 2020 framework.

\section{REFERENCES}

${ }^{1}$ R. Schoelkopf, P. Wahlgren, A. Kozhevnikov, P. Delsing, and D. Prober, Science 280, 1238 (1998).

${ }^{2}$ C. Barthel, M. Kjærgaard, J. Medford, M. Stopa, C. M. Marcus, M. P. Hanson, and A. C. Gossard, Phys. Rev. B 81, 161308 (2010).

${ }^{3}$ M. Cassidy, A. Dzurak, R. Clark, K. Petersson, I. Farrer, D. Ritchie, and C. Smith, Appl. Phys. Lett. 91, 222104 (2007).

${ }^{4}$ T. Müller, B. Küng, S. Hellmüller, P. Studerus, K. Ensslin, T. Ihn, M. Reinwald, and W. Wegscheider, Appl. Phys. Lett. 97, 202104 (2010).

${ }^{5}$ S. J. Hile, M. G. House, E. Peretz, J. Verduijn, D. Widmann, T. Kobayashi, S. Rogge, and M. Y. Simmons, Appl. Phys. Lett. 107, 093504 (2015).

${ }^{6}$ N. Ares, F. J. Schupp, A. Mavalankar, G. Rogers, J. Griffiths, G. A. C. Jones, I. Farrer, D. A. Ritchie, C. G. Smith, A. Cottet, G. A. D. Briggs, and E. A. Laird, Phys. Rev. Appl. 5, 034011 (2016)

${ }^{7}$ M.-C. Harabula, T. Hasler, G. Fülöp, M. Jung, V. Ranjan, and C. Schönenberger, Phys. Rev. Appl. 8, 054006 (2017).

${ }^{8}$ D. J. Reilly, C. M. Marcus, M. P. Hanson, and A. C. Gossard, Appl. Phys. Lett. 91, 162101 (2007).

${ }^{9}$ T. Duty, G. Johansson, K. Bladh, D. Gunnarsson, C. Wilson, and P. Delsing, Phys. Rev. Lett. 95, 206807 (2005)

${ }^{10}$ J. I. Colless, A. C. Mahoney, J. M. Hornibrook, A. C. Doherty, H. Lu, A. C. Gossard, and D. J. Reilly, Phys. Rev. Lett. 110, 046805 (2013).

${ }^{11}$ S. D. Sarma, M. Freedman, and C. Nayak, npj Quantum Inf. 1, 15001 (2015).

${ }^{12}$ D. Aasen, M. Hell, R. V. Mishmash, A. Higginbotham, J. Danon, M. Leijnse, T. S. Jespersen, J. A. Folk, C. M. Marcus, K. Flensberg, and J. Alicea, Phys. Rev. X 6, 031016 (2016).

${ }^{13}$ T. Karzig, C. Knapp, R. M. Lutchyn, P. Bonderson, M. B. Hastings, C. Nayak, J. Alicea, K. Flensberg, S. Plugge, Y. Oreg, C. M. Marcus, and M. H. Freedman, Phys. Rev. B 95, 235305 (2017).

${ }^{14}$ D. Litinski, M. S. Kesselring, J. Eisert, and F. von Oppen, Phys. Rev. X 7, 031048 (2017).

${ }^{15}$ S. Vijay, T. H. Hsieh, and L. Fu, Phys. Rev. X 5, 041038 (2015)

${ }^{16}$ A. West, B. Hensen, A. Jouan, T. Tanttu, C.-H. Yang, A. Rossi, M. F. GonzalezZalba, F. Hudson, A. Morello, D. J. Reilly, and A. S. Dzurak, Nat. Nanotechnol 14, 437-441 (2019).

${ }^{17}$ N. J. Lambert, A. A. Esmail, M. Edwards, F. A. Pollock, B. W. Lovett, and A. J. Ferguson, Appl. Phys. Lett. 109, 112603 (2016).

${ }^{18}$ G. Zheng, N. Samkharadze, M. L. Noordam, N. Kalhor, D. Brousse, A. Sammak, G. Scappucci, and L. M. K. Vandersypen, Nat. Nanotechnol. 14(8), 742-746 (2019).

${ }^{19}$ I. Ahmed, J. A. Haigh, S. Schaal, S. Barraud, Y. Zhu, C-M Lee, M. Amado, J. W. A. Robinson, A. Rossi, J. J. L. Morton, and M. F. Gonzalez-Zalba, Phys. Rev. Appl. 10, 014018 (2018).

${ }^{20}$ A. Rossi, R. Zhao, A. S. Dzurak, and M. F. Gonzalez-Zalba, Appl. Phys. Lett. 110, 212101 (2017)

${ }^{21}$ M. Urdampilleta, D. J. Niegemann, E. Chanrion, B. Jadot, C. Spence, P.-A. Mortemousque, C. Bäuerle, L. Hutin, B. Bertrand, S. Barraud, R. Maurand, M. Sanquer, X. Jehl, S. D. Franceschi, M. Vinet, and T. Meunier, Nat. Nanotechnol. 14(8), 737-741 (2019).

${ }^{22}$ S. Schaal, S. Barraud, J. J. L. Morton, and M. F. Gonzalez-Zalba, Phys. Rev. Appl. 9, 054016 (2018).

${ }^{23}$ J. Hornibrook, J. Colless, A. Mahoney, X. Croot, S. Blanvillain, H. Lu, A. Gossard, and D. Reilly, Appl. Phys. Lett. 104, 103108 (2014).

${ }^{24}$ M. F. Gonzalez-Zalba, S. Barraud, A. J. Ferguson, and A. C. Betz, Nat. Commun. 6, 6084 (2015).

${ }^{25}$ A. C. Betz, R. Wacquez, M. Vinet, X. Jehl, A. L. Saraiva, M. Sanquer, A. J. Ferguson, and M. F. Gonzalez-Zalba, Nano Lett. 15, 4622 (2015).

${ }^{26}$ A. Crippa, R. Maurand, D. Kotekar-Patil, A. Corna, H. Bohuslavskyi, A. O. Orlov, P. Fay, R. Laviéville, S. Barraud, M. Vinet, M. Sanquer, S. De Franceschi, and X. Jehl, Nano Lett. 17, 1001 (2017).

${ }^{27}$ M. F. Gonzalez-Zalba, S. N. Shevchenko, S. Barraud, J. R. Johansson, A. J. Ferguson, F. Nori, and A. C. Betz, Nano Lett. 16, 1614 (2016).

${ }^{28}$ R. Mizuta, R. M. Otxoa, A. C. Betz, and M. F. Gonzalez-Zalba, Phys. Rev. B 95, 045414 (2017).

${ }^{29}$ K. D. Petersson, C. G. Smith, D. Anderson, P. Atkinson, G. A. C. Jones, and D. A. Ritchie, Nano Lett. 10, 2789 (2010). 
${ }^{30}$ A. P. Higginbotham, T. W. Larsen, J. Yao, H. Yan, C. M. Lieber, C. M. Marcus, and F. Kuemmeth, Nano Lett. 14, 3582 (2014).

${ }^{31}$ D. de Jong, J. van Veen, L. Binci, A. Singh, P. Krogstrup, L. P. Kouwenhoven, W. Pfaff, and J. D. Watson, Phys. Rev. Appl. 11(4), 044061 (2018).

${ }^{32}$ J. van Veen, D. de Jong, L. Han, C. Prosko, P. Krogstrup, J. D. Watson, L. P. Kouwenhoven, and W. Pfaff, preprint arXiv:1903.09066 (2019).

${ }^{33}$ M. Jarratt, A. Jouan, A. Mahoney, S. Waddy, G. Gardner, S. Fallahi, M. Manfra, and D. Reilly, preprint arXiv:1903.07793 (2019).

${ }^{34}$ J. R. Petta, A. C. Johnson, C. M. Marcus, M. P. Hanson, and A. C. Gossard, Phys. Rev. Lett. 93, 186802 (2004).

${ }^{35}$ D. M. van Zanten, D. Sabonis, J. Suter, J. I. Väyrynen, T. Karzig, D. I. Pikulin, E. C. O’Farrell, D. Razmadze, K. D. Petersson, P. Krogstrup, and C. M. Marcus, preprint arXiv:1902.00797 (2019).

${ }^{36}$ P. Krogstrup, N. L. B. Ziino, W. Chang, S. M. Albrecht, M. H. Madsen, E. Johnson, J. Nygård, C. M. Marcus, and T. S. Jespersen, Nat. Mater. 14, 400 (2015).
${ }^{37}$ H. Brenning, S. Kafanov, T. Duty, S. Kubatkin, and P. Delsing, J. Appl. Phys. 100, 114321 (2006).

${ }^{38}$ S. M. Albrecht, A. P. Higginbotham, M. Madsen, F. Kuemmeth, T. S. Jespersen, J. Nygård, P. Krogstrup, and C. M. Marcus, Nature 531, 206 (2016).

${ }^{39}$ M. T. Deng, S. Vaitieknas, E. B. Hansen, J. Danon, M. Leijnse, K. Flensberg, J. Nygård, P. Krogstrup, and C. M. Marcus, Science 354, 1557 (2016).

${ }^{40}$ W. G. van der Wiel, S. De Franceschi, J. M. Elzerman, T. Fujisawa, S. Tarucha, and L. P. Kouwenhoven, Rev. Mod. Phys. 75, 1 (2002).

${ }^{41}$ N. J. Lambert, A. A. Esmail, F. A. Pollock, M. Edwards, B. W. Lovett, and A. J. Ferguson, Phys. Rev. B 95, 235413 (2017).

${ }^{42}$ M. Jung, M. D. Schrer, K. D. Petersson, and J. R. Petta, Appl. Phys. Lett. 100, 253508 (2012)

${ }^{43}$ L. P. Kouwenhoven, S. Jauhar, K. McCormick, D. Dixon, P. L. McEuen, Y. V. Nazarov, N. C. van der Vaart, and C. T. Foxon, Phys. Rev. B 50, 2019 (1994).

${ }^{44}$ S. Angus, A. Ferguson, A. Dzurak, and R. Clark, Appl. Phys. Lett. 92, 112103 (2008). 